\title{
Effect of GaP strain compensation layers on rapid thermally annealed InGaAs/GaAs quantum dot infrared photodetectors grown by metal-organic chemical-vapor deposition
}

\author{
L. Fu, ${ }^{\text {a) }}$ I. McKerracher, H. H. Tan, and C. Jagadish \\ Department of Electronic Materials Engineering, Research School of Physical Sciences and Engineering, \\ The Australian National University, Canberra 0200, Australia \\ N. Vukmirović and P. Harrison \\ School of Electronic and Electrical Engineering, University of Leeds, Leeds LS2 9JT, United Kingdom
}

(Received 8 June 2007; accepted 20 July 2007; published online 15 August 2007)

\begin{abstract}
The effect of GaP strain compensation layers was investigated on ten-layer InGaAs/GaAs quantum dot infrared photodetectors (QDIPs) grown by metal-organic chemical-vapor deposition. Compared with the normal QDIP structure, the insertion of GaP has led to a narrowed spectral linewidth and slightly improved detector performance. A more significant influence of $\mathrm{GaP}$ was observed after the structure was annealed at various temperatures. While a similar amount of wavelength tuning was obtained, the GaP QDIPs exhibited much less degradation in device characteristics with increasing annealing temperature. (C) 2007 American Institute of Physics. [DOI: 10.1063/1.2770765]
\end{abstract}

In recent years, quantum dot infrared photodetectors ${ }^{1}$ (QDIPs) based on self-organized quantum dots (QDs) have attracted extensive research interest for infrared light detection. In order to provide adequate light absorption and hence good quantum efficiency, QDIPs normally contained 10-20 multiple stacked QD layers. However, with increasing number of dot layers, the accumulation of compressive strain makes it difficult to grow stacked QD structures. For QDIPs grown by metal-organic chemical-vapor deposition (MOCVD), this issue is even more challenging due to its high growth temperatures. ${ }^{2}$ So far, only a few groups have reported good quality MOCVD grown QDIPs. ${ }^{1,2}$ Moreover, the thermally unstable stacked QD structures are also unfavorable for the realization of monolithic integration of optoelectronic devices using the intermixing technique ${ }^{3}$ which needs to be initiated through postgrowth annealing. For example, thermal annealing has been used to tune the detection wavelength of InGaAs/GaAs QDIPs. ${ }^{4,5}$ However, this is accompanied by a degradation in the responsivity ${ }^{4}$ or detectivity ${ }^{5}$ due to strain induced defect formation at high annealing temperatures.

One of the approaches to address the issue of accumulated strain is to insert a strain compensation (SC) layer. For InGaAs QDs, which are in compressive strain with respect to GaAs, a tensile GaP layer (4\% lattice mismatch) can be used. This should minimize the strain field from the previous layer and also stabilize the system during annealing, enabling us to grow or anneal a larger number of stacked layers without formation of dislocations. ${ }^{6}$ The effect of the GaP SC layer has been demonstrated to improve device performance for the QD lasers. ${ }^{7,8}$ However, for QDIPs which are normally based on even more stacked layers of QDs, its effect has not yet been reported. In this work, we study the effect of the $\mathrm{GaP}$ SC layer on the device characteristics of a ten-layer InGaAs/GaAs QDIP structure grown by MOCVD and annealed at different temperatures.

\footnotetext{
${ }^{a)}$ Electronic mail: full09@rsphysse.anu.edu.au
}

Two $n-i-n$ QDIP structures were grown on semiinsulating GaAs (001) substrates by MOCVD. QDIP1 contained ten layers of 5.7 ML undoped InGaAs (nominally $50 \%$ ) QDs, separated by $50 \mathrm{~nm}$ of GaAs barrier layers and sandwiched between two highly Si-doped top $(300 \mathrm{~nm})$ and bottom $(1000 \mathrm{~nm})$ GaAs contact layers. QDIP2 contained exactly the same layers as QDIP1 except that a thin $0.5 \mathrm{~nm}$ $\mathrm{GaP}$ layer was grown $5 \mathrm{~nm}$ below each dot layer. The growth parameters have been optimized to achieve similar uniform dot size and high dot density in the uppermost dot layer of both QDIP structures. ${ }^{2,6}$ Rapid thermal annealing (RTA) was performed on both structures at temperatures ranging from 700 to $800{ }^{\circ} \mathrm{C}$ for $30 \mathrm{~s}$ under Ar flow, which are the typical conditions used to initiate QD intermixing in InGaAs QDs without causing significant degradation in the optical properties of QDs. ${ }^{9} 10$ During annealing, the samples were sandwiched between two fresh pieces of GaAs to minimize desorption of As from the surface. Then, all the samples were fabricated into $250 \times 250 \mu \mathrm{m}^{2}$ mesa structures using standard QDIP fabrication process. ${ }^{2}$ The devices were then mounted in a liquid nitrogen cooled Dewar for characterization.

The normal incidence spectral response measurements were performed using a Nicolet Impact 400 Fourier transform infrared spectrometer and a SRS 570 low-noise current preamplifier. Figure 1(a) shows the spectral responses of the QDIPs fabricated from the as-grown samples. Similar peak positions $(\sim 210 \mathrm{meV})$ can be seen for both devices with a much narrowed spectral linewidth obtained for GaP QDIP2 (30 meV, $\Delta \lambda / \lambda \sim 15 \%)$ than QDIP1 $(68 \mathrm{meV}, \Delta \lambda / \lambda$ $\sim 32 \%$ ). To understand the effect of GaP SC on the electronic band structure of the QDIP, the effective potential profiles, energy levels, wave functions, and optical absorption cross sections were calculated using the same model described in Ref. 5. Technical details of the model are reported in Ref. 11. We have found that since the $\mathrm{GaP}$ used in our work is thin $(0.5 \mathrm{~nm})$, it has a small influence on the potential profile in QD region and energies of bound states. However, due to relatively large conduction band offset between 


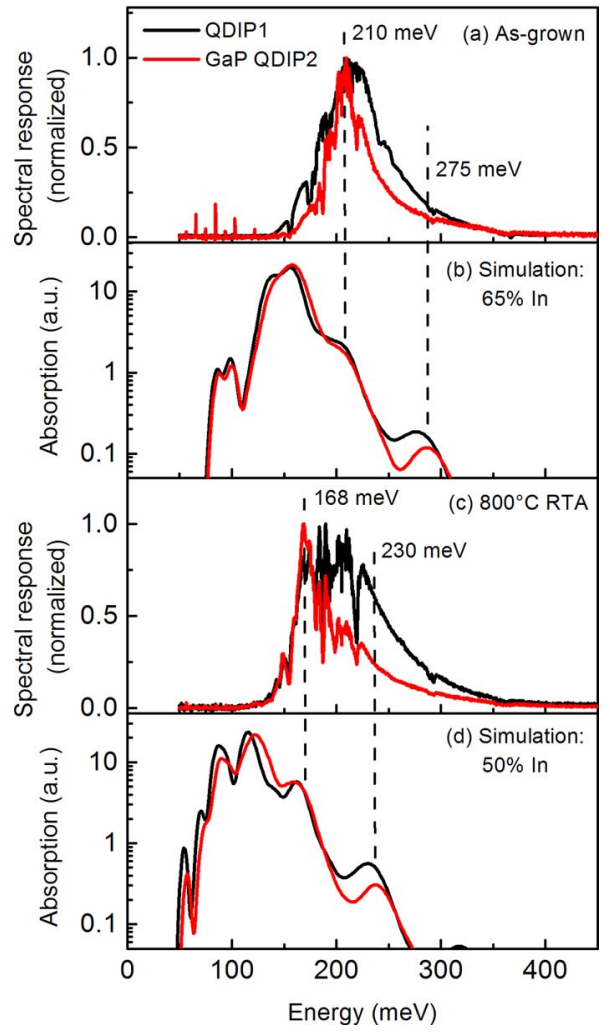

FIG. 1. (Color online) $77 \mathrm{~K}$ spectral photoresponses measured at the bias of $0.4 \mathrm{~V}$ from the as-grown (a) and $800{ }^{\circ} \mathrm{C} 30 \mathrm{~s}$ annealed (c) QDIP1 and QDIP2. The calculated spectra of optical absorption cross sections for $65 \%$ and 50\% InGaAs/GaAs QDs based on both structures are also plotted in (b) and (d), respectively.

GaAs and $\mathrm{GaP}$, the presence of GaP layer does modify the delocalized continuum states and consequently the optical absorption on the transition from ground state to continuum. This effect can be seen from the calculated absorption cross sections plotted in Fig. 1(b) based on the assumption of lens shaped QDs with the diameter of $20 \mathrm{~nm}$, height of $5 \mathrm{~nm}$, a wetting layer width of $4 \mathrm{ML}$, and In content of 0.65 [larger than the nominal value for growth due to the nonuniform In distribution for the InGaAs QDs. $]^{5}$ The peak position of both devices at $\sim 210 \mathrm{meV}$ corresponds very well with one of the peaks in the calculated optical absorption cross section spectrum under $z$-polarized radiation (growth direction). According to our calculated potential profile and energy levels, ${ }^{5}$ this peak corresponds to the first and also the strongest bound-tocontinuum $(B-C)$ transition and its intensity is similar for both QDIP1 and QDIP2. It should be noted from the simulation that another $B-C$ transition occurring at $\sim 275 \mathrm{meV}$ also has a relatively high intensity. The possible contribution of this high energy $B-C$ transition to the photoresponse of QDIP1 might be the main reason resulting in the broadness of its spectrum. In comparison, the similar $B-C$ peak of $\mathrm{GaP}$ QDIP2 is lower in intensity hence could contribute less to the broadening of the spectral linewidth.

Similar spectral narrowing effects were observed for QDIP samples with GaP after RTA. Figure 1(c) shows the photoresponse of the QDIPs which were annealed at $800{ }^{\circ} \mathrm{C}$ for $30 \mathrm{~s}$. The spectrum of QDIP1 exhibits significant further broadening, whereas that of QDIP2 is still dominated by a main peak at $\sim 168 \mathrm{meV}$, although a slightly increased intensity of its high energy "tail" can be observed. Compared to the peak position $(\sim 210 \mathrm{meV})$ of the as-grown sample, an Downloaded 24 Feb 2008 to 150.203.178.41. Redistribution subject

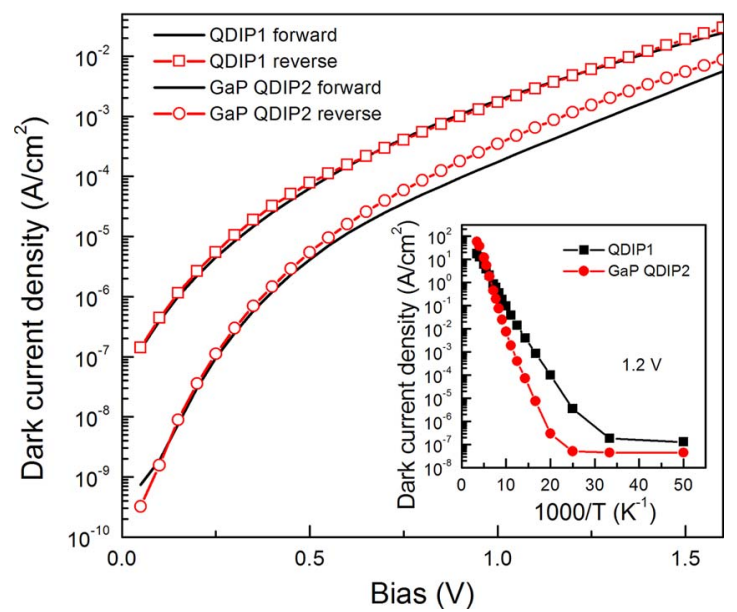

FIG. 2. (Color online) $77 \mathrm{~K} I$ - $V$ characteristics for as-grown QDIP1 and QDIP2. The inset plots the dark current density vs inverse temperature $(1000 / T)$ at the bias of $1.2 \mathrm{~V}$ for both QDIPs.

$\sim 42 \mathrm{meV}$ energy redshift is obtained. This is caused by In outdiffusion and redistribution of the QD energy states and their respective optical absorption strengths as a result of thermal interdiffusion during RTA. ${ }^{5}$ By making a simple approximation of smaller In content of $50 \%$ in the QDs (as a result of In outdiffusion), the absorption spectra for both QDIPs after $800{ }^{\circ} \mathrm{C}$ RTA are calculated and plotted in Fig. 1 (d). It can be seen that for QDIP1 the high energy $B-C$ peak was redshifted to $\sim 230 \mathrm{meV}$ [from the $\sim 275 \mathrm{meV}$ in Figs. 1 (a) and 1(b)]. Its absorption strength relative to the main low energy $B-C$ peak was also increased, thus causing a large broadening of the spectrum. For QDIP2, although a similar amount of redshift was obtained in this sample, due to the low intensity of the high energy absorption $B-C$ peak, a less change was observed in the shape of its photoresponse spectrum. This result will be useful for the development of high resolution multispectral QDIP arrays, where narrow spectral linewidth is essential.

Figure 2 shows the $77 \mathrm{~K}$ dark current density-voltage $(I-V)$ curves for the as-grown QDIPs under forward (positive bias on top contact) and reverse biases. At lower biases, the dark current density from QDIP2 is about two orders of magnitude lower than that of the QDIP1. With an increase of bias, the difference between the two devices slowly decreases to less than one order of magnitude at the bias of $\pm 1.6 \mathrm{~V}$. In comparison, the dark current density shows more distinct asymmetry for the GaP QDIP2, which mainly originates from the asymmetrically placed GaP SC layers within the GaAs barriers. The reduction of dark current mainly arises from two effects due to the presence of the GaP layers: (1) the current blocking effect and (2) the strain compensation effect. Due to their large band gap, the GaP layers form high energy barriers to the thermally emitted electrons during their transport processes to form dark current. At low biases most electrons may not gain enough energy to tunnel through despite the small thickness of the GaP layers, leading to a much reduced dark current. With increasing bias, the tunneling probability of electrons is greatly enhanced and thus, the current blocking effect of $\mathrm{GaP}$ is lessened. On the other hand, it has been shown previously ${ }^{12}$ that the incorporation of GaP layers reduces the overall strain in the stacked QD structure and thus reduces the defect density. This effect could also contribute to the reduction of the dark current in QDIPs, as can be observed from the inset of Fig. 2, which to AlP license or copyright; see http://apl.aip.org/apl/copyright.jsp 


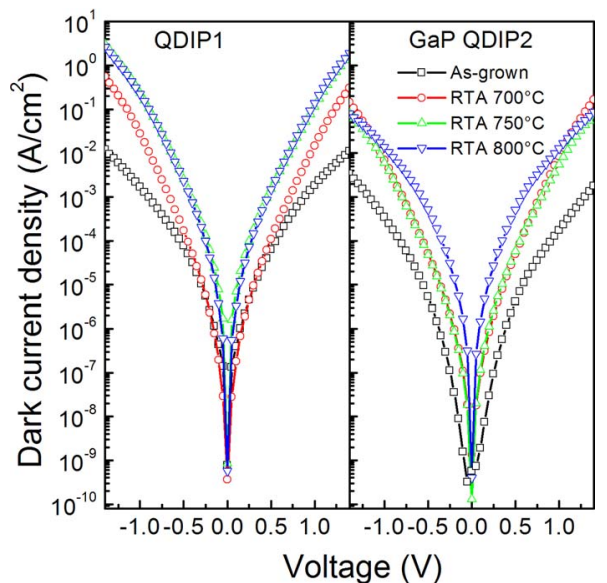

FIG. 3. (Color online) $77 \mathrm{~K} I-V$ characteristics for the QDIP1 and QDIP2 annealed at different temperatures for $30 \mathrm{~s}$.

plots the values of dark current density measured using a $10 \mathrm{~K}$ Janis closed-cycle He cryostat at temperatures ranging from 20 to $290 \mathrm{~K}$ at a bias of $1.2 \mathrm{~V}$. The curves measured from both QDIPs exhibited two different regions: a linear high temperature region due to thermionic emission and/or field-assisted tunneling; and a nonlinear low temperature region due to defect-related tunneling processes. ${ }^{13}$ In comparison to QDIP1, the decreased dark current density is obtained for QDIP2 over the entire temperature range. The slope of its thermal-limited linear region of the curve is also increased owing to the increase of the thermal activation energy for the dark current in the presence of the large band gap GaP layer. In the low temperature regime, where the dark current is dominated by the temperature-invariant process, a further reduction of the dark current density can be noted, indicating that some defect-related tunneling may be suppressed due to reduced defect formation.

The $77 \mathrm{~K} \mathrm{I-V}$ curves for the annealed QDIPs were plotted in Fig. 3. For both QDIP structures, an increasing annealing temperature caused an increase of the dark current. This has been observed in previous studies ${ }^{5}$ and ascribed to the increased ground state energy from intermixing as well as the formation of extended defects from strain relaxation and dopant-related defect complexes due to high doping in the contact layers after high temperature annealing. However, the dark current density of QDIP2 is consistently smaller than that of QDIP1 for each annealing temperature, which can be again attributed to the SC effect of the GaP which minimizes the formation of extended defects at high annealing temperatures.

The absolute peak responsivity $\left(R_{p}\right)$ and specific detectivity $\left(D^{*}\right)$ of all QDIP devices were calibrated ${ }^{2}$ using an IR-301 blackbody source (at $800{ }^{\circ} \mathrm{C}$ ) and plotted in Figure 4. For the as-grown samples, there is only a slight increase of the device responsivity and detectivity for the GaP QDIP2 despite its much smaller dark current compared to QDIP1 at the same bias. This is because that with the same transport paths for both dark current and photocurrent, although the layer of $\mathrm{GaP}$ is able to reduce the dark current, it also prevents the efficient collection of electrons to form photocurrent. The slight increase of the responsivity and detectivity may only be due to the smaller defect concentration in the

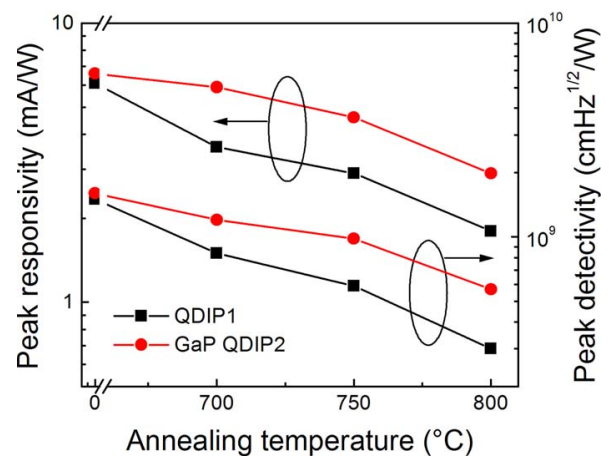

FIG. 4. (Color online) $77 \mathrm{~K}$ peak responsivity (at the bias corresponding to maximal detectivity) and detectivity of the QDIPs annealed at different temperatures for $30 \mathrm{~s}$.

as-grown GaP QDIPs. However, with increasing annealing temperature, the improvement becomes more significant, demonstrating the action of SC on stabilizing the QDIP structure and thus reducing the formation of extended defects during annealing. This is highly desirable for the application of the intermixing technique to QDIPs for wavelength tuning or optoelectronic device integration, which inevitably involves a thermal annealing process for initiation of interdiffusion.

In conclusion, we have studied the SC effect of additional GaP layers on the growth and thermal annealing of InGaAs/GaAs QDIPs. Compared with normal QDIPs (without $\mathrm{GaP}$ ), improved device performance have been achieved in the GaP QDIPs annealed under the same conditions. This is ascribed to the $\mathrm{SC}$ effect from the GaP layers, which reduces the driving force for strain relaxation and defect formation in the multilayer QDIP structures.

The authors would like to thank M. Aggett, T. Halstead, and D. Gibson for their excellent technical assistance. The financial support from Australian Research Council is also acknowledged.

${ }^{1}$ J. Jiang, S. Tsao, T. O’Sullivan, W. Zhang, H. Lim, T. Sills, K. Mi, M. Razeghi, G. J. Brown, and M. Z. Tidrow, Appl. Phys. Lett. 84, 2166 (2004), and references therein.

${ }^{2}$ L. Fu, P. Lever, K. Sears, H. H. Tan, and C. Jagadish, IEEE Electron Device Lett. 26, 628 (2005).

${ }^{3}$ Semiconductor Quantum Wells Intermixing, Optoelectronic Properties of Semiconductors and Superlattices Vol. 8, edited by E. H. Li (Gordon and Breach, Amsterdam, 2000).

${ }^{4}$ S. H. Hwang, J. C. Shin, J. D. Song, W. J. Choi, J. I. Lee, and H. Han, Microelectron. J. 36, 203 (2005).

${ }^{5}$ L. Fu, H. H. Tan, I. McKerracher, J. Wong-Leung, C. Jagadish, N. Vukmirović, and P. Harrison, J. Appl. Phys. 99, 114517 (2006).

${ }^{6}$ P. Lever, H. H. Tan, and C. Jagadish, J. Appl. Phys. 95, 5710 (2004).

${ }^{7}$ N. Nuntawong, Y. C. Xin, B. Birudavolu, P. S. Wong, S. Huang, C. P. Hains, and D. L. Huffaker, Appl. Phys. Lett. 86, 193115 (2005).

${ }^{8}$ J. Tatebayashi, N. Nuntawong, Y. C. Xin, P. S. Wong, S. H. Huang, C. P. Hains, L. F. Lester, and D. L. Huffaker, Appl. Phys. Lett. 88, 221107 (2006).

${ }^{9}$ P. Lever, H. H. Tan, C. Jagadish, P. Reece, and M. Gal, Appl. Phys. Lett. 82, 2053 (2003)

${ }^{10}$ P. Lever, H. H. Tan, and C. Jagadish, J. Appl. Phys. 96, 7544 (2004).

${ }^{11} \mathrm{~N}$. Vukmirović, Ž. Gačević, Z. Ikonić, D. Indijin, P. Harrison, and V. Milanović, Semicond. Sci. Technol. 21, 1098 (2006).

${ }^{12} \mathrm{~N}$. Nuntawong, S. Birudavolu, C. P. Hains, S. Huang, H. Xu, and D. L. Huffaker, Appl. Phys. Lett. 85, 3050 (2004).

${ }^{13}$ A. D. Stiff-Roberts, X. H. Su, S. Chakrabarti, and P. Bhattacharya, IEEE Photonics Technol. Lett. 16, 867 (2004). 